EPJ Web of Conferences 53, 02002 (2013)

DOI: $10.1051 /$ epjconf/20135302002

(C) Owned by the authors, published by EDP Sciences, 2013

\title{
Measurement of ultra-high energy cosmic rays: An experimental summary and prospects
}

\author{
M. Fukushima ${ }^{\mathrm{a}}$ \\ Institute for Cosmic Ray Research, University of Tokyo, Kashiwanoha 5-1-5, Kashiwa, Chiba \\ 277-8582, Japan
}

\begin{abstract}
Measurements of Ultra-High Energy Cosmic Rays achieved remarkable progress in the last 10 years. Physicists, gathered from around the world in the symposium UHECR-2012 held at CERN on February 13-16 2012, reported their most up-to-date observations, discussed the meaning of their findings, and identified remaining problems and future challenges in this field. This paper is a part of the symposium proceedings on the experimental summary and future prospects of the UHECR study.
\end{abstract}

\section{INTRODUCTION}

Cosmic Rays (CRs) are high energy radiations, mostly made up of protons and nuclei, arriving at the Earth isotropically from outer space. Their energies range from $10^{9} \mathrm{eV}$ to $10^{21} \mathrm{eV}$, with a steeply and monotonously decreasing flux approximately proportional to $\mathrm{E}^{-3}$. Ultra-High Energy Cosmic Rays (UHECRs) reaching an energy of $10^{20} \mathrm{eV}$ start interacting with the Cosmic Microwave Background $(\mathrm{CMB})$ during their propagation in extra-galactic space, produce nuclear $\Delta$ resonance and lose part of their energy in the decay of the $\Delta$. In 1966, just after the discovery of the CMB, Greisen, Zatsepin and Kuzmine (GZK) predicted that this interaction causes a strong suppression of the CR flux at around $10^{20} \mathrm{eV}$ (GZK cutoff) [1]. The search for this "end-point" in the CR spectrum has been one of the most important subjects of cosmic ray physics.

Cosmic Rays were discovered by V. Hess 100 years ago in 1912 using an electroscope on board a balloon reaching 5,400 m altitude. Extensive Air Showers (EAS), particle cascades caused by the high energy primary CRs interacting with an atmospheric nucleus, were identified by P. Auger in 1938 using a coincidence of Geiger counters separated by up to $150 \mathrm{~m}$ on the ground [2]. The existence of UHECRs reaching $10^{20} \mathrm{eV}$ was first reported by J. Linsley in 1962, using a network of scintillation counters deployed over an area of $8 \mathrm{~km}^{2}$ [3].

\section{ULTRA-HIGH ENERGY COSMIC RAYS}

UHECRs are extremely rare; they are detected as Extensive Air Showers reaching the ground over $100 \mathrm{~km}^{2}$ area approximately once per year. A traditional way of detecting UHECRs is by using an array of Surface Detectors (SDs) sparsely deployed on the ground. The energy and the arrival direction of the primary CR are reconstructed from the number of charged particles and their arrival times measured by the SDs. One of the largest arrays using this technique, AGASA with $100 \mathrm{~km}^{2}$ covered area using optical fiber network, reported a possible extension of the UHECR energy spectrum beyond the predicted GZK cutoff in 1998 [4].

\footnotetext{
ae-mail: fukushim@icrr.u-tokyo.ac.jp
}

This is an Open Access article distributed under the terms of the Creative Commons Attribution License 2.0, which permits unrestricted use, distribution, and reproduction in any medium, provided the original work is properly cited. 
A different method of detecting EAS was discussed by A. Chudakov, M. Oda and K. Suga in 1958-1962; they proposed to detect scintillation (fluorescence) light produced by the passage of EAS particles in the atmosphere by using an imaging telescope [5]. The first detection of EAS by air fluorescence was made in Japan by G. Tanahashi in 1968 [6], who came back from Cornell University where an intensive study of air fluorescence detection had been initiated by K. Greisen. The method was systematically developed and refined by the Fly's Eye group in Utah, and the first observation of the GZK cutoff was announced in 2008 [7] by the upgraded detector, High Resolution Fly's Eye (HiRes).

The history of EAS observation and fluorescence method was reviewed by $P$. Sokolsky ${ }^{1}$ at the beginning of UHECR-2012 (for a historical review of EAS, see also [8] by K. H. Kampert and A. A. Watson). Recent efforts of standardizing the air fluorescence yield and its dependence on the atmospheric condition were reported by B. Keilhauer.

The emerging air fluorescence method enabled:

- experimental determination of the primary CR energy by measuring the total amount of fluorescence light in the atmosphere (total absorption calorimetry), and

- statistical determination of the primary particle species, or the mass composition, by measuring the longitudinal shower development in the atmosphere $\left(\mathrm{X}_{\max }\right.$ measurement, see below).

Modern large-scale UHECR experiments, Pierre Auger Observatory (hereafter called Auger) and Telescope Array experiment (TA), are hybrid experiments employing a large SD array and a battery of Fluorescence Detectors (FDs) overlooking the SD area. The SD array has a high duty factor $(\sim 100 \%)$ and has flat acceptance above a certain threshold energy. The FD is operated only on clear, moonless nights (duty $<10 \%$ ), and its acceptance grows with the energy of CRs. The number of SD/FD coincident events (hybrid events) are limited, but they are important as they carry the largest amount of information on the produced EAS. For Auger and TA, high statistics analyses of energy spectrum and anisotropy are performed using the SD data, with its energy calibrated by the FD data using hybrid events. The mass composition is determined by using average $\mathrm{X}_{\max },\left\langle X_{\max }\right\rangle$, and its distribution width, $\mathrm{RMS}\left(\mathrm{X}_{\max }\right)$, observed by the FD, where $X_{\max }\left[\mathrm{g} / \mathrm{cm}^{2}\right]$ is defined as the slant atmospheric depth of the maximum shower development in the atmosphere.

The SD array of Auger is composed of 1600 water Cherenkov counters deployed in a triangular grid of $1.5 \mathrm{~km}$ spacing, and covers a ground area of $3000 \mathrm{~km}^{2}$. Each tank contains water in a bag with $10 \mathrm{~m}^{2}$ bottom area and $1.2 \mathrm{~m}$ height. The SD of TA is composed of 507 scintillation counters deployed in a mesh with $1.2 \mathrm{~km}$ spacing, and covers an area of $700 \mathrm{~km}^{2}$. Each TA/SD has two layers of plastic scintillators, $1.2 \mathrm{~cm}$ thick and $3 \mathrm{~m}^{2}$ large. Water Cherenkov counters and plastic scintillators are sensitive to both the Electro-Magnetic (EM) component (electrons and gammas) and hadronic component (muons) in the EAS. The Cherenkov signal in the water tank is larger for penetrating hard muons than soft electrons and gammas in the shower, thus making the Auger/SD more sensitive to the hadronic component in the EAS. The plastic scintillator of TA equally samples the charged particles, and the resultant signal from the EAS is dominated by the outnumbered electrons and photons.

The number of observed UHECRs increased by more than an order of magnitude by the new experiments, Auger and TA, and the quality of UHECR observation improved largely by the simultaneous use of the ground array (SD) and air fluorescence telescope (FD). A combination of the two experiments, Auger in Argentina (35.3 ${ }^{\circ}$ South) and TA in Utah, USA (39.4 North) now covers the entire sky for CR observations.

\footnotetext{
1 The name in italic is meant his/her contribution at the UHECR-2012 is available in the printed proceedings or in the form of presentation file at the symposium website.
} 
Table 1. UHECR-2012 Working Groups.

\begin{tabular}{|c|c|c|}
\hline Subject & Convenors & Members \\
\hline Spectrum & $\begin{array}{l}\text { B. R. Dawson } \\
\text { Y. Tsunesada } \\
\text { Y. }\end{array}$ & $\begin{array}{l}\text { T. Abu-Zayyad } 2,3 \text {, D. Ikeda }{ }^{3}, \text { D. Ivanov }{ }^{2,3} \text {, I. C. Maris }{ }^{1} \text {, } \\
\text { M. Pravdin }{ }^{4}, \text { M. Roth }{ }^{1} \text {, A. Sabourov }{ }^{4}, \text { F. Salamida }\end{array}$ \\
\hline Composition & $\begin{array}{l}\text { J. Bellido }{ }^{1}, \\
\text { J. W. Belz }\end{array}$ & $\begin{array}{l}\text { E. Barcikowski }{ }^{3} \text {, Y. Egorov }{ }^{4}, \text { S. Knurenko }{ }^{4} \text {, V. de Souza } \\
\text {, } \\
\text { Y. Tameda }^{3}, \text { Y. Tsunesada }^{3} \text {, M. Unger }\end{array}$ \\
\hline Anisotropy & $\begin{array}{l}\text { P. Sommers }{ }^{1} \text {, } \\
\text { P. Tinyakov }\end{array}$ & $\begin{array}{l}\text { O. Deligny }^{1}, \text { A. Ivanov } \\
\\
\text { H. J. Jagawa } \\
\text { He L. Timofeev }\end{array}$ \\
\hline Multi-Messenger & $\begin{array}{l}\text { M. Risse } \\
\text { G. I. Rubtsov } \\
\text { (3,4 }\end{array}$ & J. Alvarez-Muniz ${ }^{1}$, B. T. Stokes ${ }^{2,3}$ \\
\hline $\begin{array}{l}\text { Shower Simulation and } \\
\text { Hadronic Interactions }\end{array}$ & R. Engel $^{1}$ & $\begin{array}{l}\text { J. Allen }^{1} \text {, A. Castellina } \\
\text {, , K. Itakura*, K. Kasahara } \\
\text { S. Knurenko }^{3,5} \text {, S. Ostapchenko } \\
\text { T. T. Pierog } \\
\end{array}$ \\
\hline & & $\begin{array}{l}\text { 1: Auger, 2: HiRes, 3: TA, 4: Yakutsk, 5: LHCf } \\
\text { *: KEK Theory Center }\end{array}$ \\
\hline
\end{tabular}

\section{SYMPOSIUM UHECR-2012}

The symposium UHECR-2012 was held at CERN on February 13-16 $2012^{2}$ as a follow up of UHECR2010 held in Nagoya in December $2010^{3}$.

There were 230 participants from 25 countries, and 53 oral and 48 poster presentations were made. Five working groups (WGs) were formed before the symposium from members of Auger, HiRes, TA, Yakutsk air shower array (Yakutsk) and the very forward LHC experiment (LHCf). The physics subjects of the WGs are listed in Table-1, together with WG members and convenors.

The WGs addressed such questions as: how much common understanding have we reached in UHECR observations, what are the remaining differences among experiments, and what are the charges and challenges to resolve the issues. Each WG reported the results of their research in the symposium, and reports are included in the proceedings [9-13]. Two summary talks were given at the end: one for the theory and phenomenology by A. Olinto, and another for the experiment and future prospects by M. Fukushima (this report). The symposium concluded with remarks by A. A. Watson and a general discussion among all participants.

\section{ENERGY SPECTRUM}

The spectrum WG reviewed the energy spectrum of currently operating experiments, Auger, TA and Yakutsk, and of completed experiments, AGASA and HiRes. First, all spectra were fitted with broken power laws. The strong suppression of the flux, expected from the GZK process, was confirmed with good statistical significance by HiRes, TA and Auger around $10^{19.6 \pm 0.2} \mathrm{eV}$, showing a decrease of the power law index from -2.7 down to $-5.2 \sim-4.2$. This suppression was not seen in the AGASA spectrum, and Yakutsk currently has insufficient exposure in this energy region. A dip structure, or "ankle", was observed by all experiments as a change of power law index from -3.3 to -2.7 around $10^{18.7} \mathrm{eV}$.

Second, the WG examined the consistency of spectral shapes between experiments by adjusting the energy scale of each experiment to a common scale, which was taken halfway between Auger and TA. This particular choice of common energy scale, a mean of Auger and TA, reflects our belief that the energy determination by FD is experimentally better controlled than SD without relying too much on

\footnotetext{
2 http://indico.cern.ch/conferenceDisplay.py?conf Id=152124

3 http://uhecr2010.icrr.u-tokyo.ac.jp/ and http://proceedings.aip.org/resource/2/apcpcs/1367/1?
} 


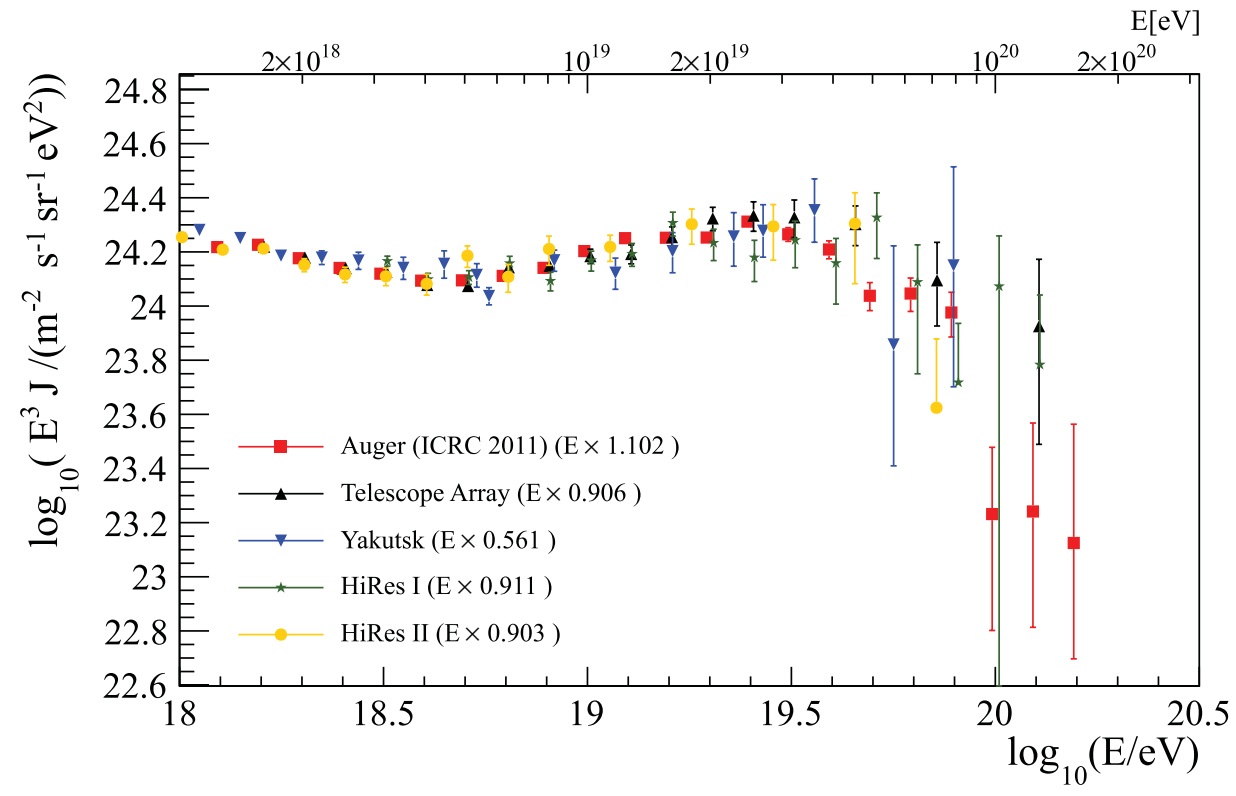

Figure 1. Re-scaled energy spectra in the form of $E^{3} \times$ flux. See spectrum WG's report for details [9].

Table 2. Energy scale factors obtained by fitting the spectrum [9].

\begin{tabular}{cccccc}
\hline Auger & TA & AGASA & Yakutsk & HiRes-I & HiRes-II \\
1.102 & 0.906 & 0.652 & 0.561 & 0.911 & 0.903 \\
\hline
\end{tabular}

the result of EAS simulations and their hadronic interaction models at UHE. Figure 1 was obtained by fitting the spectra below $10^{19.5} \mathrm{eV}$ to the common energy scale. Corresponding scale factors are listed in Table-2 [9].

The estimated energy scale uncertainties of FD measurement are 17\%, 22\% and $21 \%$ for HiRes, Auger and TA respectively, and the obtained scale factors are within these limits. The AGASA scale factor (0.652) is at the limit of consistency with the quoted systematic uncertainties of AGASA $(20 \%)$ and FD ( $20 \%$ typical). AGASA's number of events above $10^{20} \mathrm{eV}$ becomes two after the energy rescaling, and the observation of the extended spectrum beyond the GZK cutoff becomes statistically insignificant. The Yakutsk SD spectrum is calibrated by the Cherenkov light emission and its rescale factor $(0.561)$ seems to be also at the limit of consistency with the systematic uncertainty of the Cherenkov calibration $(\sim 25 \%)$.

\section{MODELS}

Observed energy spectra are interpreted by models of CR generation and propagation. In those models, the generation of CRs at the source is usually characterized by a power law spectrum and the maximum energy of acceleration. The propagation of CR causes energy losses by collisions with the CMB and Extra-galactic Background Light (EBL). In some models, effects of galactic and extra-galactic magnetic fields are considered.

Dip Model. The observed energy spectra of HiRes and TA above $10^{18} \mathrm{eV}$, in terms of the spectral shapes and their break point energies, are well explained by the GZK process; interactions of extragalactic protons with the CMB producing the "ankle" via $\mathrm{e}^{+} \mathrm{e}^{-}$pair production at around $10^{18.7} \mathrm{eV}$, and the "cutoff" via pion photo-production around $10^{19.6} \mathrm{eV}$ (see Fig. 2 by R. Aloisio). The energy $\mathrm{E}_{1 / 2}$ of 


\section{UHECR 2012}

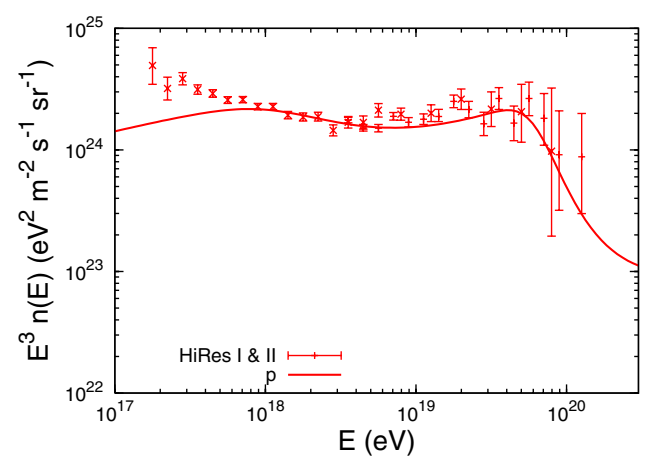

Figure 2. HiRes spectrum and the prediction of the dip model (R. Aloisio).

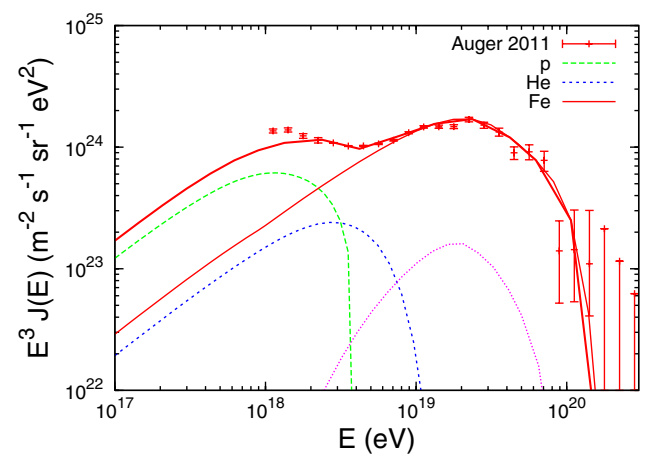

Figure 3. Auger spectrum and prediction by one of the multi-composition models (R. Aloisio).

the integrated flux is $10^{19.73 \pm 0.07} \mathrm{eV}$ for the HiRes spectrum, and agrees with the theoretical calculation of $10^{19.72} \mathrm{eV}$ (V. Berezinsky) within a systematic uncertainty of the energy scale of HiRes (17\%). This picture (dip model) is consistent with the observation of a proton-like composition above $10^{18} \mathrm{eV}$ by HiRes and TA using the $\mathrm{X}_{\max }$ measurement.

Multi-Composition Model. On the other hand, Auger, with its measured composition changing from proton to heavier nuclei in this energy range, has not associated the observed spectral features with a particular physics mechanism. A variety of models with different source composition, acceleration and propagation assumptions have been proposed to fit Auger's high statistics spectrum data, and measurements of $\left\langle X_{\max }\right\rangle$ and $\mathrm{RMS}\left(\mathrm{X}_{\max }\right)$. See Fig. 3 for example for the case of p-He-Fe multicomposition by $R$. Aloisio. Although these models often contain CR acceleration limits at the origin as important ingredients, it is generally conceived that the photo-disintegration of iron nuclei by the CMB is the dominant cause creating the high energy flux suppression. The effect of photo-disintegration on nuclei was already mentioned in the original GZK papers. The ankle may be explained in these models as the acceleration limit of extra-galactic protons, or of the galactic heavy components corresponding to a galactic to extra-galactic transition of CRs.

The following experimental challenges are identified to pin down the correct physics model of UHECR spectrum:

- Energy scale uncertainty in FD: The flux suppression by the GZK process, whether it is for proton or for iron, is absolute in energy scale, and the parameter such as $E_{1 / 2}$ is relatively insensitive to the choice of the spectral index at the source and the cosmological evolution of CRs. Note that, between Auger and TA, there is a significant difference in the energy scale; 

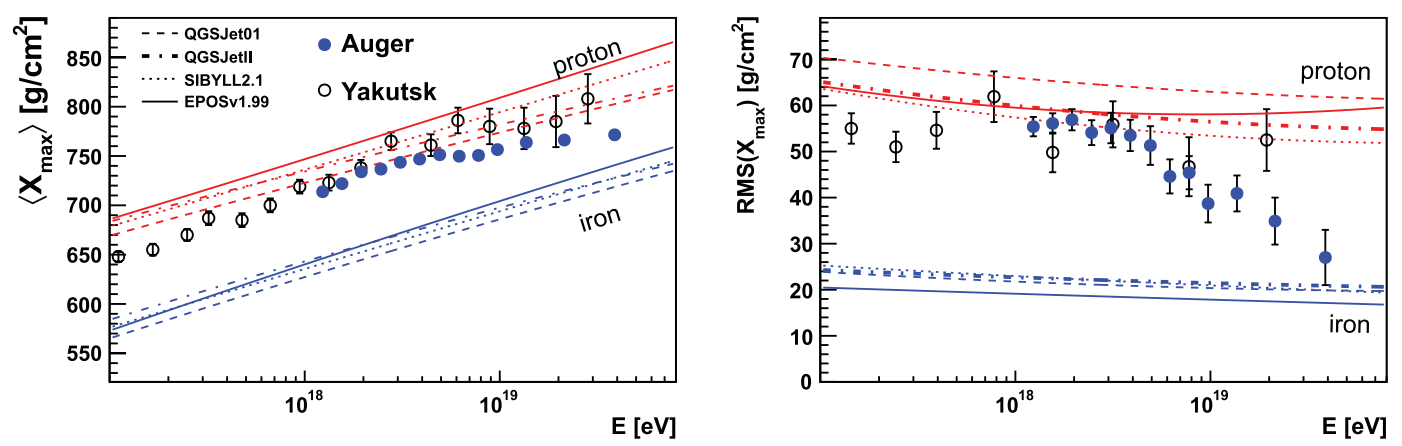

Figure 4. The energy dependence of $\left\langle X_{\max }\right\rangle$ for Auger and Yakutsk (left). The measured $\left\langle X_{\max }\right\rangle$ for Auger and Yakutsk has negligible analysis biases, and is directly comparable with the simulation (red and blue lines). The energy dependence of RMS $\left(\mathrm{X}_{\max }\right)$ for Auger and Yakutsk is shown on the right. The RMS $\left(\mathrm{X}_{\max }\right)$ of Auger is defined as the r.m.s. of the $\mathrm{X}_{\max }$ deviations. The experimental resolution estimated from the simulation is subtracted in quadrature. The highest energy bin of Yakutsk contains only 3 events.

Auger is $\sim 18 \%$ lower than TA in the rescaling of energy spectra, and Auger's cutoff breakpoint energy is $\sim 46 \%$ lower than that of TA $\left(10^{19.68} \mathrm{eV}\right.$ for TA and $10^{19.41} \mathrm{eV}$ for Auger. See [9]).

- Energy linearity in SD: The calibration of SD energy is made only by the limited sample of $\mathrm{SD} / \mathrm{FD}$ coincident events, i.e. the direct calibration is limited to energies below the onset of the high-energy cutoff. The extrapolation to higher energies relies on the validity of the Constant Intensity Cut (CIC) method for Auger, and Monte Carlo air shower simulation for TA, both of which require independent experimental verification.

- Mass Composition: The mass composition plays a key role in distinguishing different models. The change of $\left\langle X_{\max }\right\rangle$ also directly affects the energy determination. Meaningful measurement of $\mathrm{X}_{\max }$ is now limited to $10^{18.0} \mathrm{eV}-10^{19.5} \mathrm{eV}$. An extension of composition measurement to both higher and lower energy regions is important.

- Around the cutoff and above: The spectral shape above $10^{19.5} \mathrm{eV}$ may be a convolution of the GZK cutoff and the acceleration limit of UHECRs. The cutoff shape also carries information on the composition as seen in Figs. 2 and 3. Above $10^{19.5} \mathrm{eV}$, the agreement of spectral shapes among experiments is not as satisfactory as the lower energy region (see Fig. 1). In order to determine the exact shape of the cutoff above $10^{19.5} \mathrm{eV}$, measurements with higher statistics and less systematic error are essential.

\section{MASS COMPOSITION}

The primary mass composition of UHECRs is determined from the $\mathrm{X}_{\max }$ measurement. The $\mathrm{X}_{\max }$ can be determined for each FD event, but the mass composition is usually determined only statistically by comparing the $\left\langle X_{\max }\right\rangle$ and $\mathrm{RMS}\left(\mathrm{X}_{\max }\right)$ with the expectation from the EAS simulation. This is due to the large event-by-event fluctuation of $\mathrm{X}_{\max }$, particularly for proton events, and the limited accuracy of $\mathrm{X}_{\max }$ measurement. The most straight-forward way of measuring $X_{\max }$ is by imaging the EAS development in the atmosphere using the FD, which has been adopted by HiRes, Auger and TA. There are less direct way of measuring the Cherenkov light distribution on the ground (by Yakutsk) and muon detection on the ground (by Auger/SD).

Measurements of $\left\langle X_{\max }\right\rangle$ and $\mathrm{RMS}\left(\mathrm{X}_{\max }\right)$ are compiled by the composition WG [10]. They are shown in Fig. 4 for Auger and Yakutsk, in Figs. 5 and 6 for HiRes, in Fig. 7 for TA (preliminary) together with the results of air shower simulations for protons and iron. The different measurements are 


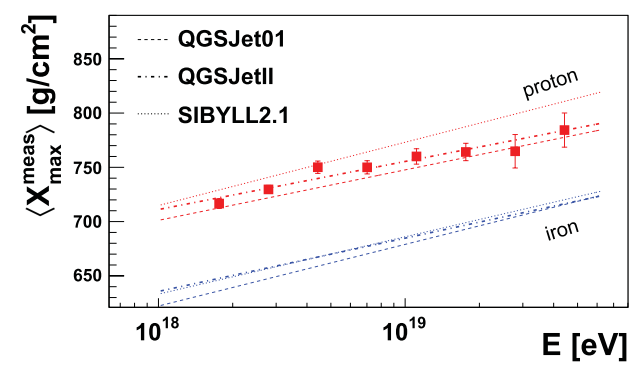

Figure 5. The energy dependence of $\left\langle X_{\max }\right\rangle$ for HiRes. The measured $\left\langle X_{\max }\right\rangle$ is compared with the simulation (lines) with analysis biases included both for the measurement and simulation.

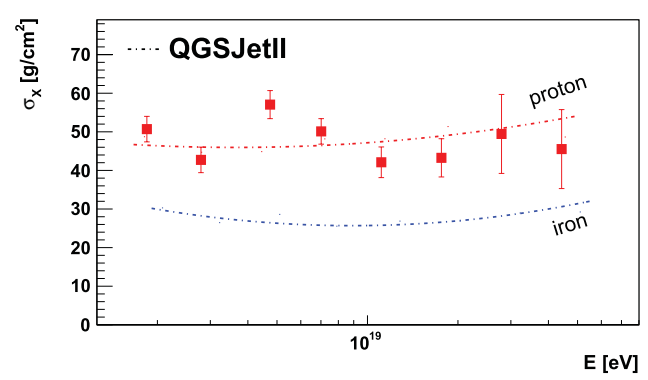

Figure 6. The energy dependence of $\operatorname{RMS}\left(\mathrm{X}_{\max }\right)$ for HiRes. The $\operatorname{RMS}\left(\mathrm{X}_{\max }\right)$ is defined as the $\sigma$ obtained by fitting the $X_{\max }$ distribution with a Gaussian truncated at $2 \sigma$. The experimental resolution is included for both the measurement and simulation.

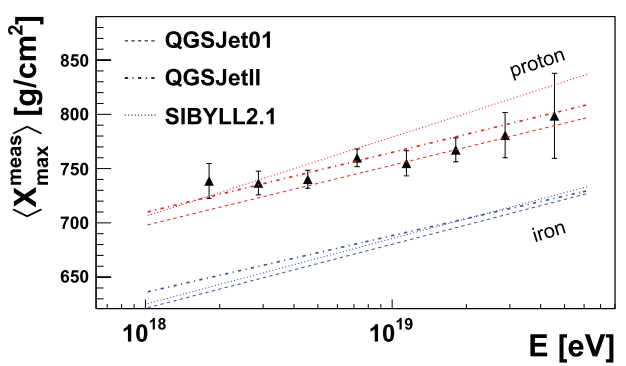

Figure 7. The energy dependence of $\left\langle X_{\max }\right\rangle$ for TA (preliminary). The measured $\left\langle X_{\max }\right\rangle$ is compared with the simulation (lines) with analysis biases included for both the measurement and simulation.

not overlaid in one plot because each experiment treats, either includes or removes, analysis biases and resolution effects differently, and no direct comparison of measured values is adequate.

As seen in the plots, all experiments agree that the light component (proton) dominates in the energy region below the ankle for $10^{18.2} \mathrm{eV}-10^{18.5} \mathrm{eV}$. Above $10^{18.5} \mathrm{eV}$, Auger's measurement suggests a change of composition from light to heavier, reaching almost iron at $10^{19.5} \mathrm{eV}$. This is seen in both $\left\langle X_{\max }\right\rangle$ and $\mathrm{RMS}\left(\mathrm{X}_{\max }\right)$, but is more pronounced in $\mathrm{RMS}\left(\mathrm{X}_{\max }\right)$. On the other hand, no clear indication of the composition change is seen for the $\left\langle X_{\max }\right\rangle$ of HiRes, TA and Yakutsk up approximately to $10^{19.3} \mathrm{eV}$.

Distinctions between Auger and HiRes/TA/Auger is obviously that Auger covers the southern sky, and HiRes/TA/Yakutsk cover the northern sky. Among other important distinctions are event detection 
topologies and data analysis methods; Auger uses hybrid events using FD and SD (muon based) in coincidence, and HiRes and TA use stereo FD events using two FDs (EM energy based) in coincidence. In the data analysis, Auger uses tight acceptance cuts to minimize the analysis bias, whereas HiRes and TA use looser acceptance cuts to accept more events and use elaborate simulations to estimate the acceptance bias. The composition WG scrutinized the analysis methods of Auger, HiRes and TA in order to understand whether the analysis method is responsible for the difference.

In order to guarantee good measurements of $\mathrm{X}_{\max }$, all experiments apply the so-called bracket cut for the acceptance. This cut requires that the $\mathrm{X}_{\max }$ of individual events be observed in the Field of View (FoV) of the FD telescope. The tight bracket condition, however, causes certain bias in $\left\langle X_{\max }\right\rangle$ and $\mathrm{RMS}\left(\mathrm{X}_{\max }\right)$ because, for a given event geometry, we do not know a priori how many unbracketed events exist outside the FoV.

HiRes and TA developed an elaborate EAS event simulation, analyzed the generated MC events using the same calibration and analysis programs as the data, and the data-MC agreement was checked. The event reconstruction was made by the inverse-MC method using the same parameter set for the data and MC. The data observables, including $\left\langle X_{\max }\right\rangle$ and $\mathrm{RMS}\left(\mathrm{X}_{\max }\right)$ with its energy dependence, are well reproduced by the pure proton primary using the QGSJET-II interaction model, and this is the basis for the claim of an unchanged proton component by HiRes and TA. The estimated acceptance bias of TA is less than $15 \mathrm{~g} / \mathrm{cm}^{2}$ for QGSJET-II proton above $10^{18.6} \mathrm{eV}$, and is negligible for QGSJET-II iron. The reconstruction bias, defined as the difference between the real (thrown) $\mathrm{X}_{\max }$ and the reconstructed $\mathrm{X}_{\max }$, is approximately $10 \mathrm{~g} / \mathrm{cm}^{2}$ independent of the energy and the composition.

Auger's $\mathrm{X}_{\max }$ analysis requires tighter condition for the acceptance such that an event is accepted only if not only the observed $\mathrm{X}_{\max }$ but also an entire range of the probable $\mathrm{X}_{\max }$ of that event geometry (and energy) is included in the FoV. The range of accepted $\mathrm{X}_{\max }$ is determined by checking that the resultant $\left\langle X_{\max }\right\rangle$ is not influenced by the FoV cuts [10]. This method is tuned to minimize the $\mathrm{X}_{\max }$ acceptance bias, at the cost of fewer accepted events; the tight FoV cut reduces the accepted events of Auger by half for energies above $10^{18.2} \mathrm{eV}$. The performance of the analysis was estimated by using the MC; the combined (reconstruction + acceptance) $X_{\max }$ bias is smaller than $4 \mathrm{~g} / \mathrm{cm}^{2}$, and the $X_{\max }$ resolution is $20-25 \mathrm{~g} / \mathrm{cm}^{2}$.

The composition WG made numerous checks on the analysis results, and the data-MC agreement was examined for many observables as reported in [10]. In short, no particular flaw of analysis, or definite clue for the resolution of the difference was identified so far. The WG is now proceeding to the next step to analyze the Auger data with TA's looser cuts, to analyze the TA data with Auger's tight cut, in order to see whether the result is unchanged. Work is also in progress to generate and analyze MC event sets having the same $\mathrm{X}_{\max }$ distribution as observed by Auger, and pass them through the analysis chain of TA and HiRes.

The results of $\left\langle X_{\max }\right\rangle$ from different experiments (or analysis methods) may be compared by converting the $\left\langle X_{\max }\right\rangle$ into $\langle\ln \mathrm{A}\rangle[10]$, as it is known that the change of $\mathrm{X}_{\max }$ is approximately proportional to $\ln \mathrm{A}$ at a fixed CR energy, where A is the atomic number of the primary CR. The composition WG calculated the value of $\langle\ln \mathrm{A}\rangle$ for each experiment by proportionating the difference of the measured $\mathrm{X}_{\max }$ between the expected $\mathrm{X}_{\max }$ for protons and iron. The result obtained by using QGSJET-II is shown in Fig. 8. The shaded area indicates the systematic errors of each experiment; $12 \mathrm{~g} / \mathrm{cm}^{2}$ for Auger and TA, $20 \mathrm{~g} / \mathrm{cm}^{2}$ for Yakutsk, $6 \mathrm{~g} / \mathrm{cm}^{2}$ for HiRes. It is seen that HiRes and Auger give different results and are not compatible for energies above $10^{18.5} \mathrm{eV}$. The results of TA and Yakutsk partially overlap both with HiRes and Auger. A comparison using the SIBYLL model makes the difference between Auger and HiRes smaller, but they do not overlap above $10^{18.5} \mathrm{eV}$.

It should be noted that the statistics of Auger is higher than all other experiments combined, even with the tight acceptance cut ${ }^{4}$. The WG report demonstrated that the energy dependence of Auger's

\footnotetext{
4 The number of events above $10^{19} \mathrm{eV}$ is 452 for Auger, 123 for HiRes, 67 for TA and 22 for Yakutsk.
} 


\section{UHECR 2012}

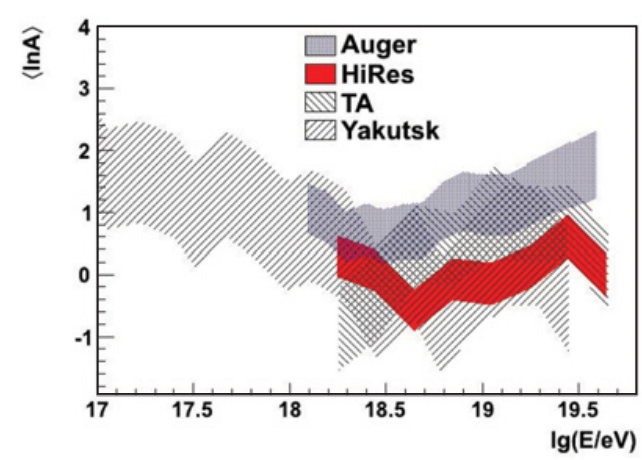

Figure 8. The energy dependence of average composition 〈ln A〉 using QGSJET-II. The shaded area indicates systematic uncertainties of each experiment. The energies are rescaled as in the case for the spectrum comparison.

$\langle\ln A\rangle$ is statistically not consistent with a constant value of $\langle\ln A\rangle$, whereas the same plots by other experiments are statistically consistent with both constant and changing values of $\langle\ln A\rangle$ with respect to $\log (\mathrm{E})$ (see Fig. 12 of [10]). More events (preferably $\times 3$ or more) in future, particularly in the northern hemisphere, are needed to conclude definitively whether the composition stays light as the energy increases, or turns heavy as in the case of Auger in the southern hemisphere. In addition, on-going improvements of EAS simulation with better models and newly available collider data (see Sect. 9) are expected to contribute a lot to predict the $\left\langle X_{\max }\right\rangle, \mathrm{RMS}\left(\mathrm{X}_{\max }\right)$ and its energy development in a more reliable way. The composition WG will continue its activity in future.

Finally, the FD and SD hardware and their calibration needs due attention for the $\mathrm{X}_{\max }$ comparison. This becomes important when the comparison of analyses does not solve the problem. HiRes, TA and Auger use different optics, PMT matrix and signal recording methods for the FD telescope. The atmospheric models and its attenuation effects are different for each experimental site. S. Ogio, in his talk of future plans [20], proposed to exchange a set of SDs and FDs between Auger and TA sites, and to make a simultaneous observation of the same UHECR event. This will be very effective in excavating systematics in the detector and analysis, if any important one exists.

\section{ANISOTROPY}

It is expected that the magnetic deflections of UHECRs by the galactic and extra-galactic magnetic fields decrease down to a few degrees for energies larger than $10^{20} \mathrm{eV}$, and a correlation between the UHECR arrival direction and the source "star" would be showing up. This correlation would be particularly distinct if the source astrophysical objects are limited within the GZK horizon ( $100 \mathrm{Mpc})$. In 2007, Auger published a correlation of UHECR $\left(\mathrm{E}>57 \mathrm{EeV}^{5}\right)$ arrival directions and nearby AGNs $(\mathrm{z}<0.018$, or within $75 \mathrm{Mpc}$ ) in the Veron-Cetty and Veron (VCV) catalog, 12th edition within a separation of $3.1^{\circ}$ [14]. The result was updated in the Beijing ICRC conference and its skymap and time development diagram are shown in Figs. 9 and 10. The rate of correlated UHECR events presented in Beijing decreased from $61 \%$ to $33 \%$ but it is still $\sim 3.0 \sigma(\mathrm{P}=0.006)$ away from a chance correlation. A concentration of correlated events in the vicinity of Cen A was reported in [15], but the significance did not increase despite higher statistics [16].

HiRes searched for a correlation in the northern hemisphere but did not find any. TA searched for correlations in the recent SD data sample with zenith angles less than $45^{\circ}$, and found 11 events are

\footnotetext{
$557 \mathrm{EeV}=10^{19.76} \mathrm{eV}, \mathrm{EeV}=10^{18} \mathrm{eV}$. The original publication had a threshold energy of $56 \mathrm{EeV}$. It was later stated to be equivalent to $57 \mathrm{EeV}$ in the updated energy scale.
} 


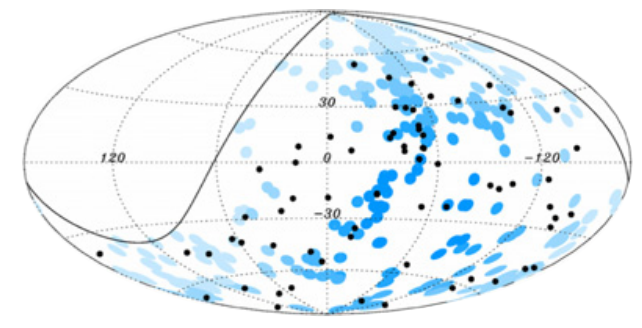

Figure 9. Arrival directions of UHECRs $(\mathrm{E}>57 \mathrm{EeV})$ in the southern sky observed by Auger. A correlation with nearby AGNs $(\mathrm{z}<0.018)$ was suggested.

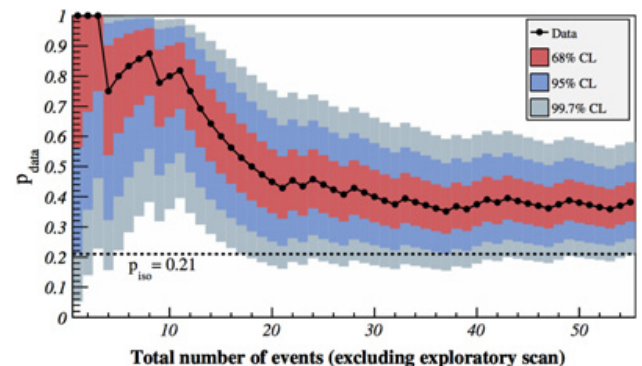

Figure 10. Time development of Auger AGN correlation. The ratio of AGN-correlated events over the total is plotted. The dashed line corresponds to the ratio expected from the accidental coincidence.

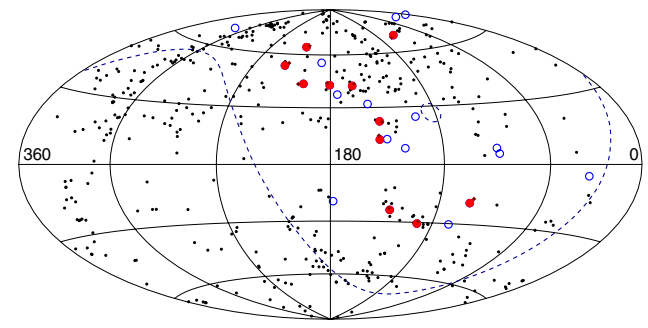

Figure 11. Arrival directions of UHECRs in the northern sky observed by TA. The same correlation conditions as in Fig. 9 are applied.

correlated from the total of 25 events under the same conditions as the Auger AGN correlation [17] ${ }^{6}$. The number of correlation expected from the uniform and random set of 25 events is 5.9, or the observed "correlation" is more than $2 \sigma(\mathrm{P}=0.02)$ away from the accidental correlation. Both HiRes and TA did not optimize the search parameters and used the same conditions as Auger in order to stay free from the search bias. The skymap of TA and the time development of the correlation are shown in Figs. 11 and 12.

Auto-correlations among UHECR events are searched for by HiRes, Auger and TA but no positive results have been reported. A global harmonic analysis was performed by Auger, and correlations with the Large Scale Structure (LSS) of galaxies have been searched for by HiRes, Auger and TA, but the results are so far consistent with both the isotropy and the LSS.

\footnotetext{
6 The energy scales of TA/HiRes may be $\sim 20 \%$ larger than that of Auger as seen in section 4, and the effective energy threshold for the search may be different by this amount.
} 


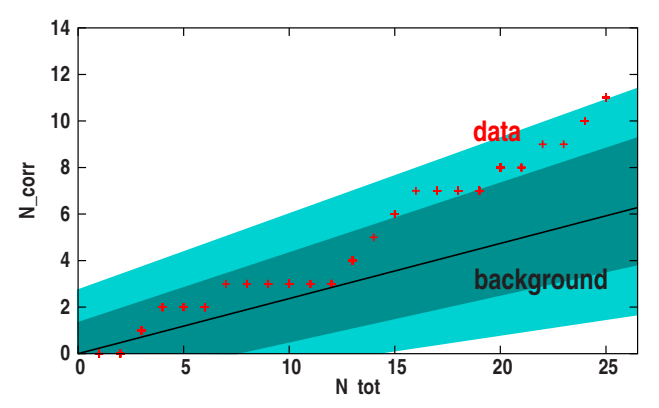

Figure 12. Time development of AGN correlation of TA. The number of AGN-correlated events is plotted. The solid line corresponds to the number expected from the accidental coincidence.

The anisotropy WG summarized that, despite a large effort spent on the search for anisotropy and correlation, no clear signal has so far been established with certainty [11]. Various hints, however, are emerging from the observations of Auger and TA, and they should be closely followed in coming days. The prospects and challenges by the anisotropy WG are reiterated as follows:

- Composition: Expectation for correlation obviously depends on the actual composition, or the atomic number, of the primary UHECRs. It should be noted that above $10^{19.7} \mathrm{eV}$ where the correlations with AGNs and LSS are expected, no direct measurement of composition by $\mathrm{X}_{\max }$ exists. Discrimination power for composition on an event-by-event basis would be a great asset for the future anisotropy search.

- Large Exposure: The next 5 years will be an exciting time when some of the hints of anisotropy seen by Auger and TA will be clarified with more statistics. In order to fully test the observed anisotropy, or to find new kind of anisotropy and characterize its cause, one order of magnitude larger exposure is needed by the next generation observatory.

- Energy Assignment: An accurate energy assignment of each event is essential for making energy cuts for trans-GZK anisotropy and also for large-scale anisotropy studies at EeV energies. The energy scale error (of FD) is expected to be improved from the present $\sim 20 \%$ down to $\sim 10 \%$, matching the energy resolution (of SD) at the highest energy region. The present angular resolution of $\sim 1^{\circ}$ may be sufficient for the future anisotropy study ${ }^{7}$.

- Full-sky coverage: There are many advantages to operate an observatory with full-sky coverage; all possible point sources would be exposed, and the structure of anisotropy with minimal exposure distortion could be achieved. Besides, with full-sky coverage, the methods of spherical harmonics and multipole analysis could be applied without additional assumptions on the anisotropy behavior in the uncovered region of the sky. Unified analysis of southern and northern skies are common interests of existing groups; such analysis was initiated using the Auger and TA data by the anisotropy WG, and is being pursued now.

- Wide Energy Coverage: Currently preferred models of UHECR (see Sect. 5) suggest that cosmic rays are extra-galactic above $10^{18} \mathrm{eV}$, but the scenario of transition from galactic to extra-galactic below $10^{18} \mathrm{eV}$ is not established, and the predictions of models differ (see Berezinsky). Understanding this transition is an important step forward to provide a complete picture for origins of UHECRs. The transition should be signaled by the composition change (from heavy to light), spectrum deformation (second knee?) and the energy-dependent anisotropy as hinted by the phase change in the harmonic analysis [11] of Auger. Such low-

\footnotetext{
${ }^{7}$ For TA/SD events, the energy resolution is $13 \%$ and the angular resolution is $1.1^{\circ}$ for $\mathrm{E}>57 \mathrm{EeV}$ by simulation studies. Other experiments have similar numbers.
} 


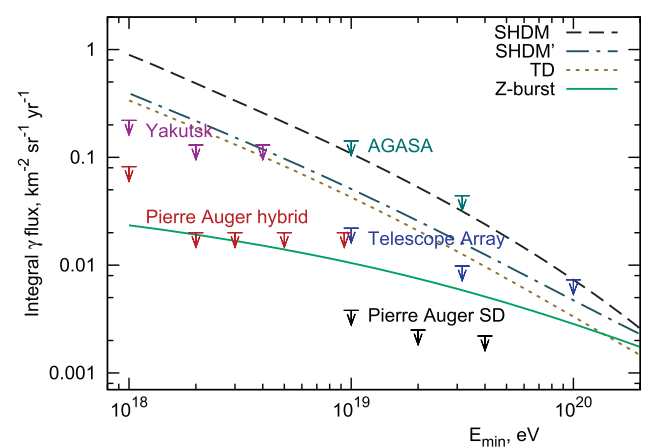

Figure 13. Experimental limits placed on the UHE photon flux and expectation from several top-down acceleration models of UHECRs.

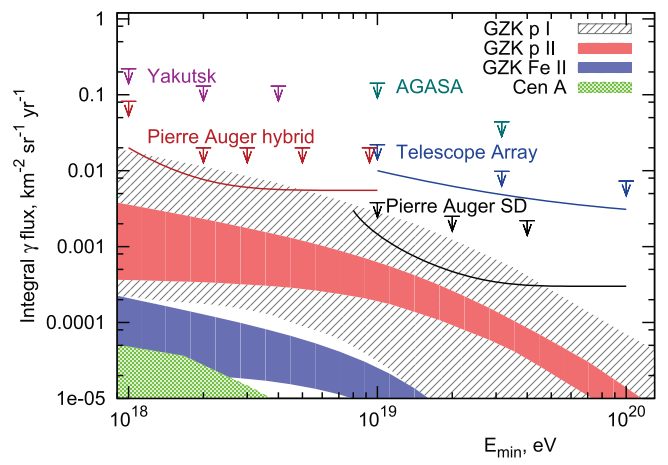

Figure 14. Experimental limits placed on the UHE photon flux. Expected limits by 2015 are indicated by lines. The shaded region corresponds to the flux predicted by several cosmogenic models [12].

energy extensions are proceeding well as HEAT and AMIGA in Auger and TALE in TA to supply high quality, high statistics data down to $10^{16.5} \mathrm{eV}$ in the near future.

\section{MULTI-MESSENGER (UHE GAMMAS AND NEUTRINOS)}

Any top-down model of UHECR origin expects abundant UHE $\gamma$ rays and neutrinos as a decay product of $\pi$ mesons. The GZK process also produces UHE gammas and neutrinos from pion decays of the excited nucleon resonance. No such UHE cosmic $\gamma$ 's and $v$ 's have been observed so far by the UHECR experiments.

Limits on the flux of UHE $\gamma$ 's by Auger, TA and Yakutsk already place strong constraints on the top-down models as seen in Fig. 13 [12]. The multi-messenger WG further compiled the present (and "anticipated" by 2015) experimental upper limits and compared them with the expected flux from cosmogenic (GZK) production models. They are reproduced here in Figs. 14 and 15.

The expected sensitivities for UHE $\gamma$ 's in Fig. 14 tells us that the next generation UHECR detector with one order of magnitude larger exposure than the present Auger and TA has a significant chance to detect cosmogenic $\gamma$ rays, provided the primary composition is proton. The iron primary is expected to give an order of magnitude smaller flux than the proton primary. The UHECR detectors planned for future, such as the nGO and JEM/EUSO (section 10) may be the only detectors to observe UHE cosmogenic $\gamma$ rays. 


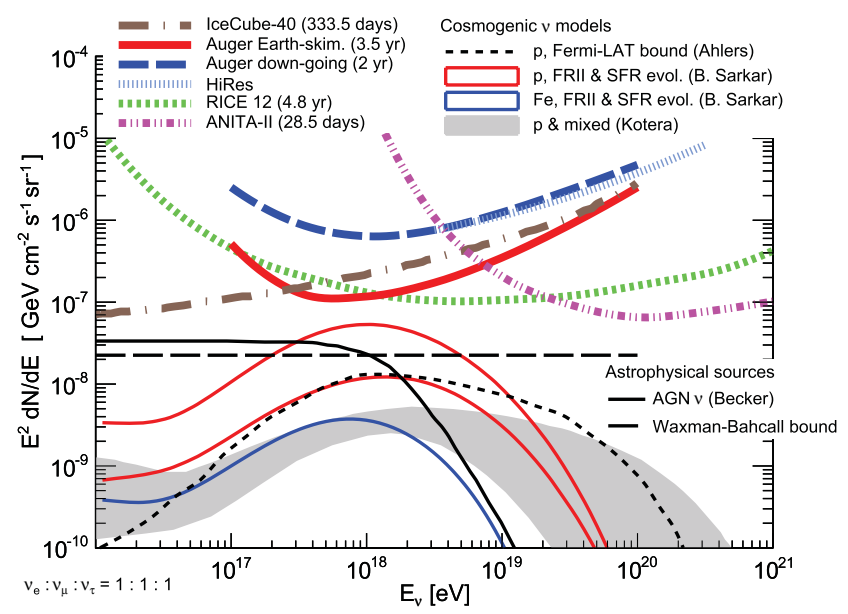

Figure 15. Experimental limits placed on the UHE neutrino flux. Expected fluxes from several cosmogenic models are shown by the shaded region and black lines. For details, see WG's report [12].

For the case of cosmogenic $v$ 's, as seen in Fig 15, the recently completed IceCube (IC-86) will have a good chance of detecting UHE $v$ 's after $\sim 5$ years of running in the region of $10^{17} \mathrm{eV}-10^{19} \mathrm{eV}$, for the case of primary protons. The south pole radio experiments, ANITA by balloon and RICE in the ice, already contribute to set upper limits for energies greater than $10^{19} \mathrm{eV}$. Future huge ground array nGO with 10 times larger acceptance than the present Auger, covers the UHE $v$ detection in the region of $10^{17} \mathrm{eV}-10^{20} \mathrm{eV}$, and in case if detected, it would give most precise information on the energy and arrival direction, and possibly the species $(e, \mu, \tau)$ of the UHE neutrinos. The JEM/EUSO will have the largest sensitivity but its energy acceptance is limited above $10^{19.5} \mathrm{eV}$. The iron primary or the mixed composition may reduce the detection possibility of cosmogenic neutrinos by roughly an order of magnitude.

\section{HADRONIC INTERACTION AND AS SIMULATION}

Despite the success of determining the UHECR energy by the new air fluorescence technique, and identifying the GZK traces in the energy spectrum, understanding the EAS itself is still incomplete and unsatisfactory. The problem is demonstrated in the following discrepancies between the EAS observation and the predictions of EAS simulation:

- Number of Muons: The number of muons measured on the ground, which is a tracer of hadronic energies in the EAS, is up to $\sim 100 \%$ larger than that predicted by the standard air shower simulation (see A. Yushkov and G. Rodriguez). It is certain that hadronic interaction models at UHE, as well as the nuclear effect, is responsible for this. Incomplete hadronic interaction database at lower energies may also be contributing to this discrepancy.

- Longitudinal Shower Development: Different interaction models give different longitudinal development and $\mathrm{X}_{\max }$ distribution. The prediction of $\left\langle X_{\max }\right\rangle, \mathrm{RMS}\left(\mathrm{X}_{\max }\right)$ and their energy development (elongation rate) differ among models. The measured $\left\langle X_{\max }\right\rangle$ and $\mathrm{RMS}\left(\mathrm{X}_{\max }\right)$ are not well reproduced by the simulations. Cases of good agreement exist, such as the HiRes/TA measurements and QGSJET/proton simulation, but this does not sufficiently prove that "QGSJET" is the correct hadronic interaction model at UHE and the proton is the unique primary "composition" of UHECR. It is interesting to note, however, that existing model predictions for iron have little differences for $\left\langle X_{\max }\right\rangle$, RMS $\left(X_{\max }\right)$ and its energy development. 
- Lateral Shower Development: The energy of UHECR events measured by the TA/SD is 27\% higher than the energy measured by TA/FD. The AGASA/SD (plastic scintillator) gives higher energy than TA/SD, but only by about $9 \%$. The systematic uncertainty of energy measurement is $21 \%$ for TA/FD and $\sim 20 \%$ for TA/SD and AGASA, meaning that the difference is at the limit of both systematics. This difference means that the same EM energy measured 600 $800 \mathrm{~m}$ away from the shower core by TA/SD and AGASA/SD is larger than the EM energy measured by the FD near the shower axis, suggesting the data tends to have larger lateral development than the simulation predicts.

The advent of the LHC is opening up a possibility to improve this situation drastically. The LHC maximum beam energy is scheduled to be $7.0 \mathrm{TeV}$, corresponding to a $10^{17} \mathrm{eV}$ cosmic ray proton colliding with a proton target at rest. Hadronic and nuclear interactions at UHEs can be studied now at accelerators and the results can be fed back to the hadronic interaction models. Two very forward experiments at the LHC are particularly relevant. One is the LHCf measuring the spectrum of neutral particles $\left(\gamma, \pi^{\circ}\right.$, and neutron) at $0^{\circ}$. The LHCf is dedicated for cosmic ray related studies. Another is the TOTEM experiment to measure total and (in-)elastic hadronic cross sections in the very forward region. It also offers valuable data to determine the development of EAS in the atmosphere.

In addition to the heavy ion collision data available from the RHIC the LHC is foreseeing protonlead collisions next year, which will contribute to the understanding of the nuclear effect at UHE. This situation was reviewed and discussed by Y. Itow, T. Sako, T. Pierog, R. Engel and other symposium contributors. A report of hadronic interaction and simulation WG is also available [13].

Using newly available data from accelerator experiments, an update of hadronic interaction models are on the way for QGSJET (from v03 to v04) and EPOS (from v1.99 to -vLHC). T. Pierog reported the status of tuning with highlights on the following observables.

- Total Cross Section: The total inelastic cross section determines the average depth of the first interaction of UHECRs in the atmosphere, and affects the speed of shower development and its fluctuation. The newly available data from TOTEM is being used for the tuning.

- Multiplicity: This parameter, as well as the inelasticity, affects the speed of shower development, and the number of muons on the ground. The data from CMS and ALICE at large angles (small pseudo-rapidity) are being used for the tuning.

- Inelasticity, or Energy Spectrum at Forward Region: The higher the leading particle energy, the slower the shower development expected. The data from CMS, ALICE and PHENIX are checked with the predictions of simulations. The $\mathrm{p}$ and $\mathrm{p}_{\mathrm{T}}$ distribution of $\gamma$ 's and $\pi^{\circ}$ 's obtained by LHCf will be useful for this tuning.

- (anti-)Nucleon Production at Very Forward Angles: The forward-produced nucleon is effective to maintain hadronic energies in the longitudinal shower development, thus affecting the muon rate at the ground level. The neutron production rate being analyzed by the LHCf experiment is eagerly waited.

Auger recently published a measurement of the $\mathrm{p}$-air total cross section using the $\mathrm{X}_{\max }$ tail distribution around $10^{18} \mathrm{eV}-10^{18.5} \mathrm{eV}$ where the dominant $\mathrm{CR}$ composition is considered to be proton [18]. In future, with the progress of understanding the CR composition, a possible appearance of the new phase of hadronic interactions and/or unknown collective nuclear effects at UHE may be revealed in the data of EAS development. When it happens, it would become a good case for the bi-lateral contribution between the CR physics at UHE and elementary particle physics.

It should be noted that the energy region above $10^{17} \mathrm{eV}$ is where we expect the galactic to extragalactic transition of cosmic rays to take place (see Sect. 7). On-going low energy extensions of HEAT/AMIGA and TALE will be soon producing high statistics spectrum and composition data in this energy range, where the EAS simulation is being calibrated directly by the precision accelerator data. 


\section{UHECR 2012}

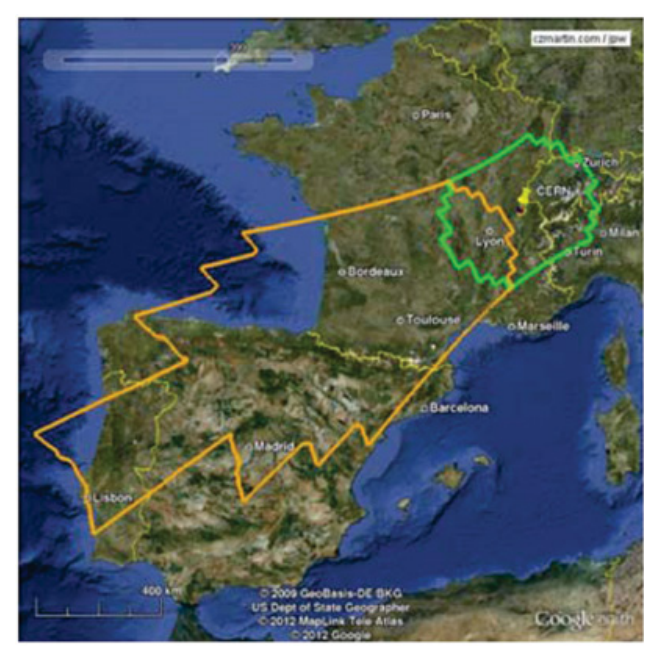

Figure 16. Footprint of the JEM-EUSO FoV projected on the ground: nadir mode (green) and tilted mode (orange) are shown [19].

\section{PROSPECTS}

A discussion on the future global facilities and the associated R\&D were made in the symposium, keeping in mind the rapid progress of UHECR research and the still remaining experimental challenges as described above.

- Next Generation Ground Observatory: Three conceptual designs for the next generation Ground Observatory with huge aperture (called nGO in this report) were presented by $A$. Letessier-Selvon, P. Privitera and S. Ogio. Astrophysical objectives of nGO were briefed by E. Parizot, and interdisciplinary science was discussed by L. Wiencke.

- Space Observatory: Observation of UHECRs from space was reviewed by T. Ebisuzaki, and the mission, performance and the prototype test of JEM/EUSO were presented by $A$. Santangelo, M. Bertaina and M. Casolino.

- R\&D for Radio Detection: Radio detection of UHECRs at MHz range was discussed by $O$. Scholten and A. M. van den Berg. It was extensively studied by LOPES and CODALEMA, and now by AERA at the Auger site. $C$. Williams reported on the GHz radio measurement by the accelerator beam (MAYBE). Field tests for detecting the air shower radio signal at the Auger site (AMBER, EASIER and MIDAS), and at KASCADE-Grande (CROME) were reported by P. Facal and R. Smida. J. Belz reported a plan of bistatic radar detection of UHECRs (TARA) at the TA site.

A majority of the designs and R\&D presented in the symposium concentrated on realizing a huge detection area, which is an order of magnitude larger than that of Auger. New generation experiments aim to collect $\sim 1,000$ events each year in the trans-GZK energy region $\left(\mathrm{E}>10^{19.7} \mathrm{eV}\right)$ to identify the astrophysical origins of UHECRs. Their features may be compared as follows:

Ground vs. Space: The SDs of Auger and TA measure the UHECRs with an angular resolution of $\sim 1.0^{\circ}$ and an energy resolution of $\sim 10 \%$ at $10^{20} \mathrm{eV}$. The nGO plans to extend the aperture maintaining the same level of resolution for trans-GZK events. This can be achieved by tuning the SD detector size and the separation using the presently available UHECR samples. A typical aperture of nGO is $55,000 \mathrm{~km}^{2} \mathrm{sr}(S$. Ogio). For Auger and TA, the determination of composition by FD is statistical and is limited to hybrid events (duty $<10 \%$ ). For nGO, a separate measurement of the EM energy and 


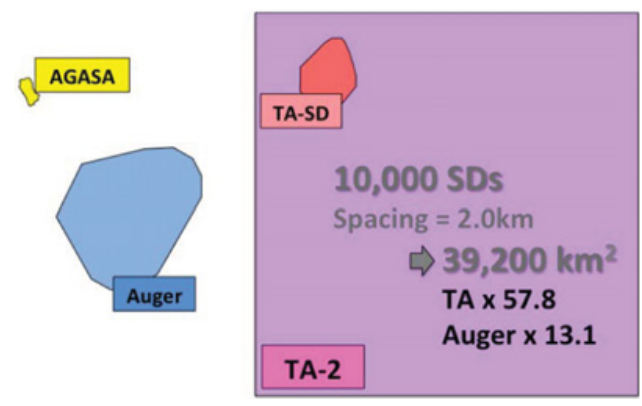

Figure 17. The covered ground areas of past, present and future ground arrays. The proposed huge array by S.Ogio [20] has 10,000 TA-type plastic scintillator SDs deployed with $2.0 \mathrm{~km}$ spacing and covers an area of $39.2 \mathrm{~km}^{2}$.

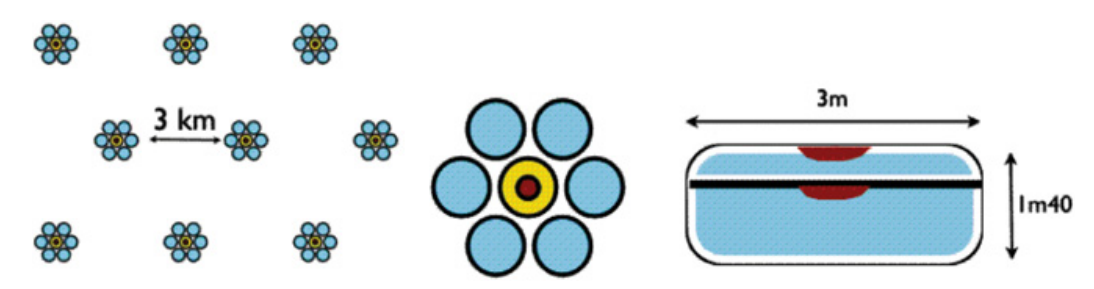

Figure 18. A possible configuration of the multi-component UHECR Observatory proposed by A. Letessier-Selvon [21]. It uses updated water tank SDs of Auger-type. Vertical segmentation of the tank serves for the EM calorimetry (upper thinner layer) and the muon counting (lower part).

the number of muons particularly at each nGO/SD will give us a handle to determine the composition event-by-event (A. Letessier-Selvon).

The JEM/EUSO aims at $2.5^{\circ}$ and $30 \%$ resolution at $10^{20} \mathrm{eV}$. The $\mathrm{X}_{\max }$ resolution is $120 \mathrm{~g} / \mathrm{cm}^{2}$ and allows a separation of $\gamma$ 's and $\nu$ 's from protons and iron. The energy threshold is $\sim 10^{19.5} \mathrm{eV}$. A large aperture of $100,000 \mathrm{~km}^{2} \mathrm{sr}$ (after $20 \%$ duty factor applied) is obtained by a single telescope looking down on the earth's atmosphere from the International Space Station (ISS) at a height of $400 \mathrm{~km}$. A tilt mode will increase the aperture to $350,000 \mathrm{~km}^{2}$ sr but with a sacrifice in performance. The JEM/EUSO design is tuned to search for astrophysical origins in the GZK horizon, and the all sky-coverage in the ISS orbit is advantageous for this purpose.

Fluorescence vs. Radio: A composition measurement by FD continues to be indispensable in the future nGO. The entire nGO area may be covered by an array of wide angle FD telescopes deployed in a mesh of $\sim 20 \mathrm{~km}$. Given that the event geometry is reconstructed using the nGO/SD information, only the time development of $\mathrm{nGO} / \mathrm{FD}$ signal is sufficient to reconstruct the EAS longitudinal development. Such information can be collected by a small number of PMTs with much larger pixel size than that of Auger and TA, thus drastically reducing the cost for construction and maintenance (P. Privitera). The trigger capability, signal to noise ratio and resolution of $\mathrm{X}_{\max }$ reconstruction are being studied for this scheme.

When a radio detector is integrated in $\mathrm{nGO}$, it would supply information of shower longitudinal development with high duty $(\sim 100 \%)$, little atmospheric or weather correction and at low cost. Such a radio detector may be used in the nGO in place of the now standard FD telescopes. Many laboratory and field measurements have been performed to detect $\mathrm{MHz}, \mathrm{GHz}$ and radio echo signals from EAS. The existence of MHz signal is firmly established by earlier experiments of LOPES, CODALEMA and others, and it is known that the signal emission is forward peaked and has a clear correlation with the geo-magnetic field. The GHz signal is expected to have an isotropic and un-polarized emission from the molecular bremsstrahlung, but so far the observed GHz signals by CROME and EASIER seem forward 
peaked, and not identified as the molecular bremsstrahlung signal. The forward peak, in both $\mathrm{MHz}$ and $\mathrm{GHz}$ cases, means the footprint of the signal on the ground is small and the radio measurement would be costly, requiring many antennae to cover the ground. The TARA radio echo test experiment is now set up on the TA site. It consists of a $54 \mathrm{MHz}, 40 \mathrm{~kW}$ television transmitter illuminating the air above $\mathrm{TA}$, and a set of radio receivers on the far side of TA, aiming to detect a forward scattered radio signal in coincidence with the TA measurement.

So far, no proven radio method to replace the air fluorescence measurement is found, nevertheless, the R\&D of radio signal detection continues in order to understand the nature of signal generation, and with its application for the $\mathrm{X}_{\max }$ measurement in mind.

Tank vs. Scintillator: The Cherenkov signal of Auger water tanks is larger for penetrating muons than soft electrons and gammas in the shower, thus making the Auger/SD more sensitive to a change of composition with energy. Muons are also a good measure to investigate hadronic interactions in the shower development.

The plastic scintillator of TA equally samples the muon and electron, and the resultant signal is dominated by the outnumbered electrons, or the EM energy. Since the bulk of the primary energy of UHECRs is transferred to the EM energy, separate measurement of the EM and muon components in the nGO/SD would allow us to take both advantages: a good linearity in the primary energy measurement by the EM component, and a good sensitivity to the primary composition (and hadronic interaction) by the muon counting.

For UHE $\gamma$ and $v$ detection, a high profile of the water tank would allow a detection of very inclined EAS down to lower energies than thin plastic scintillators can achieve. The contrary may be true for the detection of UHE $\gamma$ ray interactions which contain much fewer muons than normal EAS of the same primary energy.

In order to exchange ideas and experiences for the next generation detector, it was agreed to form a new working group for future projects. The JEM/EUSO group also expressed an interest to join the working group. The design of the next generation detector becomes more sensible and realistic when the current issues of primary composition and the energy scale/linearity are understood. An exchange of analysis method, calibration, simulation, obtained data and eventually the FD/SD detectors may be considered among experiments to attack this formidable problem. It is encouraging, as a first attempt of many others to be considered, Auger and TA initiated a photometric and optical calibration of FDs by flying a common standard light source in Utah and Malargue by using a GPS controlled octo-copter. The first flight at TA was completed in October 2012, and the next flight at Auger site is planned in November.

\section{SUMMARY AND ACKNOWLEDGEMENT}

The scene of UHECR is rapidly evolving. We just settled a long lasting, difficult experimental question on the energy spectrum of extra galactic cosmic rays; Yes, there is a "cutoff" at $10^{19.6} \mathrm{eV}$ and "ankle" at $10^{18.7} \mathrm{eV}$. The accuracy of energy measurement is about $20 \%$ now by the new air fluorescence method, and it is steadily improving. In the northern hemisphere, a constant particle composition of proton is measured above $10^{18.2} \mathrm{eV}$, whereas the composition is changing from proton to iron in the southern hemisphere. We eagerly examine our detectors and data analysis to know whether this difference is the nature or an experimental artifact. Calibration of air shower simulation is proceeding with the LHC and other accelerator data. We do hope it will shed light on the interpretation of $\mathrm{X}_{\max }$ data. No UHE gammas and neutrinos were detected, and the top-down acceleration of UHECR is strongly disfavored. Limits of astrophysical acceleration of UHECR is not seen, or may have been seen, depending on the composition result and its model interpretation. A transition of cosmic ray sources from galactic to extra-galactic may be identified in the near future by low energy extensions of present experiments (HEAT/AMIGA and TALE). Signatures of UHECR sources come and go, though certain levels of anisotropy seem to be an unavoidable consequence of the present observations. By JEM/EUSO, space may become part of our 
work field in the near future. It will seek to identify UHECR origins in all sky in the trans-GZK region. The R\&D and design of nGO, a new Ground Observatory, is proceeding. It will accumulate 1,000 events of well observed UHECRs each year to definitively solve existing questions on the UHECR, and to explore a new field of physics in the universe. So, be patient (P. Privitera), and stay tuned.

This summary report is based on the work of UHECR-2012 contributors and working groups. I have to regretfully admit the introduction of their works to this report is very incomplete. For more details and to understand complicated points, I encourage readers to consult the original papers in the same proceedings. To make this summary report, I am largely indebted for the work of contributors and WGs, but also feel responsible for my views expressed in this report, and any mistake, bias and inaccuracy appearing in this report. The UHECR-2012 was a very active and enjoyable symposium. High scientific activities and good spirits of the participants, and the symposium organizers, are truly appreciated.

\section{References}

[1] K. Greisen, Phys. Rev. Lett. 16 (1966) 748; G. T. Zatsepin and V. A. Kuz'min, Zh. Eksp. Teor. Fiz. 4 (1966) 114 [JETP Lett. 4 (1966) 78]

[2] P. Auger, R. Maze and T. Grivet-Meyer: Academie des Sciences, Paris 206 (1938) 1721

[3] J. Linsley, Phys. Rev. Lett. 10 (1963) 146

[4] M. Takeda et al., Phys. Rev. Lett. 81 (1998) 1163

[5] Norikura Symposium Report, Uchusen Kenkyu 3-5 (1958) 449; K. Suga: Proc. 5th Interamerican Symposium on Cosmic Rays, La Paz, eds. I. Escobar et al. 2 (1962) XLIX-1-5

[6] T. Hara, F. Ishikawa, G. Tanahashi et al., Acta Phys. Acad. Sci. Hung. 29-53 (1970) 369

[7] R. U. Abbasi et al., Phys. Rev. Lett. 100 (2008) 101101

[8] K. H. Kampert and A. A. Watson, Eur. Phys. J. H37 (2012) 359

[9] "The Energy Spectrum of Cosmic Rays at the Highest Energies" by the spectrum WG in these proceedings

[10] "Mass Composition Working Group Report" by the Mass Composition WG in these proceedings

[11] "Review of the Anisotropy Working Group at UHECR-2012" by the Anisotropy WG in these proceedings

[12] "Review of the Multimessenger Working Group at UHECR-2012" by the Multi-messenger WG in these proceedings

[13] Report on the Simulation and Hadronic Interaction WG in these proceedings

[14] J. Abraham et al., Science 318 (2007) 062002

[15] P. Abreu et al., Astropart. Phys. 34 (2010) 314

[16] K. H. Kampert, Proceedings of the 32nd International Cosmic Ray Conference, Aug. 11-18, 2011, Beijing, arXiv:1207.4823

[17] T. Abu-Zayyad et al., Astrophys. J. 757 (2012) 26

[18] P. Abreu et al., PRL 109 (2012) 062002

[19] "Space-based Observation of the Extensive Airshowers", T. Ebisuzaki, these proceedings

[20] "Future plans for the Telescope Array experiment", S. Ogio, these proceedings

[21] "The need for a multicomponent UHECR Observatory", A. Letessier-Selvon, these proceedings 